\title{
DAIRY SUPPLY CHAIN SYSTEM BASED ON BLOCKCHAIN TECHNOLOGY
}

\author{
Shivaprasad More \\ Assistant Professor \\ Sanjay Ghodawat University \\ Kolhapur, Maharashtra, India \\ Mrunal Chougule, Shagufta Darwajkar, Anushka Dhawan, Poonam Konnuri, Bhumi Patel \\ Sanjay Ghodawat University \\ Kolhapur, (MS), India
}

\begin{abstract}
Blockchain an advent technology especially used for securing transactions. Mostly, the blockchain is used in the banking domain especially. But nowadays the concept has been wide. Nowadays, the banking domain is not an exception, it's widely used in supply chain management also. The past decade has the importance of blockchain in supply chain integration. The knowledge management and supply chain integration ensure high profit and sustainability in the business. To fully understand the blockchain application in the food supply management or product supply management technique, cryptography is applicable. The application can be enhanced by IoT or RFID cards. This paper focuses mainly on the food supply industry or product supply management system. This paper enhances the scope of the blockchain by identifying key variables, the implications of blockchain in the food industry. This represents the present, current challenges, and future research scope in the field of blockchain.
\end{abstract}

Keywords- Blockchain, Internet-of-Things, food supply chain, Enterprise resource planning.

\section{INTRODUCTION}

Since past years the interest in Blockchain technology has been increased. There are many companies and research institutions that have great interest in the field of blockchain. Technology such as financial, industrial, and social sectors have increased scope in the blockchain. The blockchain is an early stage of development. It has potential for real-life commercial applications such as the banking domain, financial domain, etc. The innovation in the blockchain system architecture, applications, and business concepts is happening at the speed. It is often characterized by decentralized and open source development.

Blockchain technology has an open-source distributed database. This database is used to store the transaction details. Blockchain allows two parties to make the transaction directly using a duplicate link ledger and this is called the blockchain. This makes a transaction transparent than those provided by the centralized system services. So the transactions are executed without depending on the explicit trust of the third party. So, by blockchain, there is no need to trust third-party services. So, by using this technology, transparency can be improved and in turn, the supply chain system can be improved.

\section{LITERATURE REVIEW}

\section{Traceability in supply chain}

There are several approaches to be applied for supply chain traceability. The approach can be from traditional recording on papers to sophisticated and advanced technologies. There are also cutting-edge technologies for traceability in supply chain systems. The paper-based approach is highly favored for the food supply chain. This approach is in less developed counties. E.g. Berman.

The majority of farmers in China are not even interested to record documents and exchange with the wholesalers that are buying their products. They are alone participating in a complex computer-based system.

Academic research is also happening in the RFIDbased traceability system. There are many studies in recent years on different food products. This technology is also 
applied by a lot of global leading supermarket chains such as Walmart, TESCO, etc. There are a lot of advantages of RFID over the barcode system. The advantages include speed, scalability, and accuracy. In the developed countries, the barcodes are still used to support or even replace RFID systems at times. The QR code is an up-gradation from linear barcode because it provides higher data storage. The QR code also provides encryption capabilities. It is also environment friendly.

\section{Blockchain for food traceability}

The blockchain concept was first introduced in 2008 . The invention has happened with the digital currency called bitcoins. This is done by Satoshi. In November 2015. Alexis Bateman [2] believed that blockchain would be a real technological option to address the challenge of mining and managing information across the supply chain. This is implemented due to the lack of standardization. The company named "Provenance" presented to the public plan to develop a project using blockchain. this project is to develop a track of the origin of fishes. This is to respond to the demand and supply chain transparency.

\section{LIMITATIONS OF EXISTING SYSTEM}

The existing system is mostly used in the banking domain for transaction security. The product supply management system is mostly used with QR codes. The QR code system has the limitation of data storage. There are other systems such as RFID card readers. These cards are electronic means. So damage in the RFID card fails the system. The RFID cards also need to add an extra budget, there is a limitation for handling it.
IV. PROPOSED SYSTEM MODEL

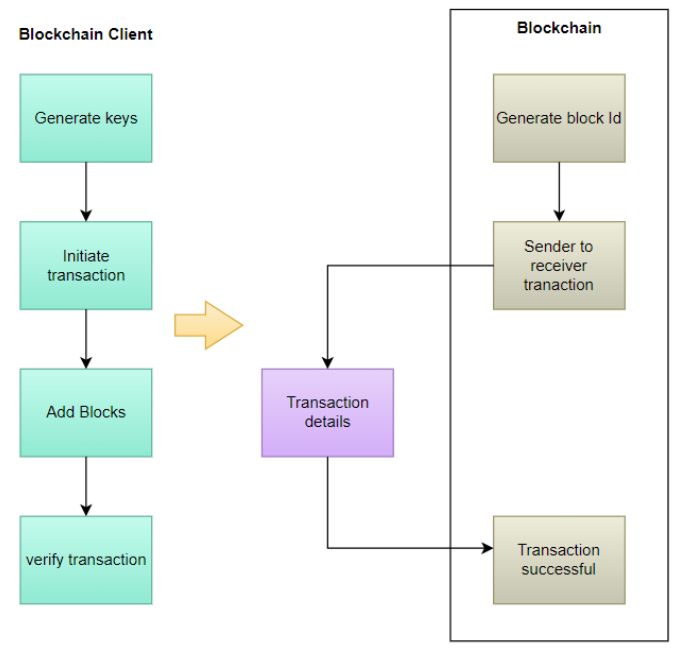

Our model provides a system having blockchain and blockchain clients. The blockchain client has the authority to view transactions of any particular block. The blocks can be created multiple times and the transaction can happen in between two blocks. The public key and private keys are created randomly. The public key of the sender, the private key of a sender, and the public key of a recipient, the product details can be added to initiate a transaction. Once the transaction is initiated it's displayed at the client-side and also at the particular node side. The blockchain client can also view all the transactions. Once the transaction is successful, its initiated as a successful transaction.

\section{IMPLEMENTATION AND ANALYSIS}

Blockchain technology uses hash encryption to secure the data, relying mainly on the SHA256 algorithm to secure the information. The address of the sender (public key), the receiver's address, the transaction, and his/her private key details are transmitted via the SHA256 algorithm. The encrypted information, called hash encryption, is transmitted across the world and added to the Blockchain after verification. The SHA256 algorithm makes it almost impossible to hack the hash encryption, which in turn simplifies the sender and receiver's authentication. 


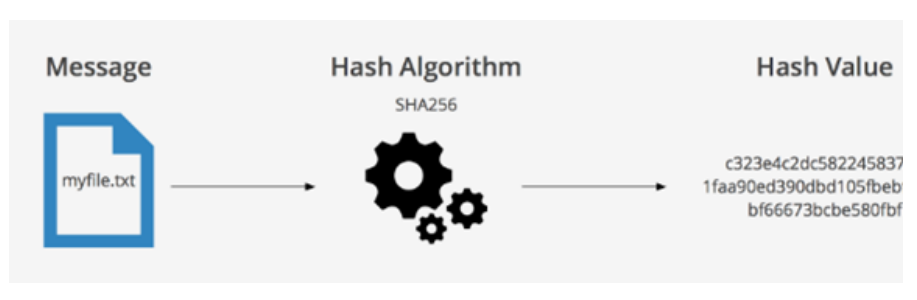

\section{METHODOLOGY}

The methodology includes the generation of keys. The keys are nothing but private and public keys. These are called cryptography keys. By using any cryptographic algorithm such as SHA or RSA, the keys are generated. There is a shared ledger that has transaction details between two blocks. The ledger has the block address and the amount or the product details. There is a peer-to-peer network and that is used for sharing the ledger. The ledger has the transaction details. The computation is nothing but to store the transaction and records of the network. The process of the transaction contains hash encryption. There is proof of work that is going to check the previous hash. There is the concept of mining is used to check which block is to be selected for the transaction.

\section{IMPLEMENTATION DETAILS}

The public and private keys are created by using the RSA algorithm. The random function is used for creating the values. The keys are 256 bits. The hashing methods are used here for the blockchains. There is a method for the calculation of the previous hash, transaction details, and hash address of the block. This is included in the proof of work. The implementation details include the following points:

1. Cryptographic keys:

The cryptographic keys consists of two keys- public key and private key. These keys perform the transaction between two parties. Each node is given one of each a private key and a public key which produce a digital identity reference.

2. A peer-to-peer network containing a shared ledger:

The blockchain technology uses digital signature with the peer-to-peer network. There are many authorities that use digital signature to reach a common agreement(consensus) on the transactions. So simply putting, Blockchain users use the cryptographic keys to perform digital transactions between two parties over the peer-to-peer network.

3. A means of computing, to store the transactions and records of the network

4. Process of Transaction:
The most fundamental feature of Blockchain Technology is the way it authorizes the transactions. Lets take a example, two parties use a public and private key respectively, the party $\mathrm{A}$ attachs the information of transaction to the public key of party $\mathrm{B}$ and this information is stored in a block.

This block holds relevant information such as a digital signature, a timestamp, etc., but the should not contain specifications of the parties doing the transaction. Then, the block is sent all across the network's nodes and if the right party uses the its private key and it matches with the block, the transaction is then successful.

5. Proof of Work: POW is a consensus algorithm, through proof of work all the peers in the blockchain network reach a common agreement.

Previous Hash: This hash address detects the previous block.

Transaction Details: Details of all the transactions that need to take place.

Nonce: An arbitrary number given in cryptography to differentiate the block's hash address.

6. Hash Address of the Block: All the above (i.e., preceding hash, transaction details, and nonce) are transmitted through a hashing algorithm. This gives an output containing a 256-bit, 64-character length value.

\section{RESULT AND COMPARATIVE STUDY}

This paper has the research objective of investigating the possibility of blockchain implementation. The cornerstone in the development of traceability solutions avoids food frauds or product frauds. In the earlier research, the blockchain has main use in the transaction. So in the banking domain, the blockchain has mostly used. But nowadays, the blockchain has a wide scope, The blockchain can be used in dairy supply products to encrypt the nutrients values. It can be also used for price encryption. In the previous results, the blockchain has limitations about the transaction. But blockchain can be used for product details encryption and security purposes in supply chain management systems. The below graph indicates the processing overhead comparison of the both selected system and the proposed system. 


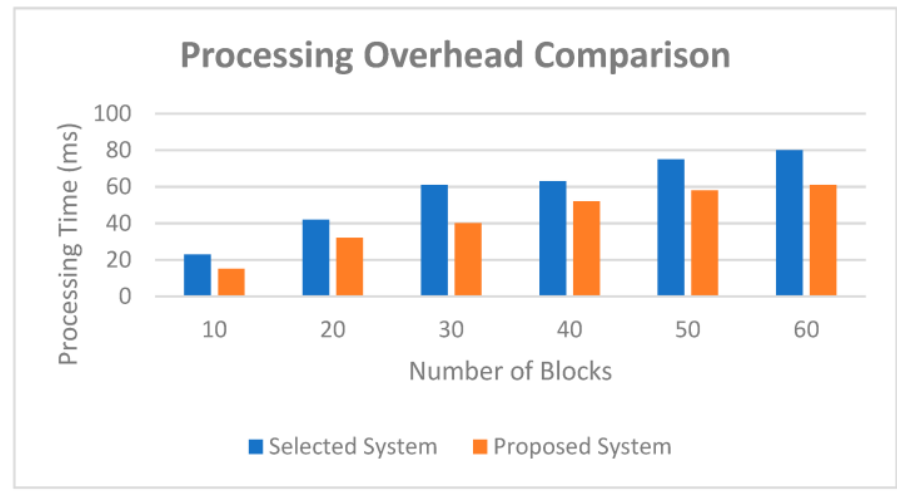

\section{CONCLUSION}

It is useful to understand blockchains in the context of product supply management system, but you should not assume that all blockchain ecosystems need system mechanisms such as proof of work, longest chain rule,

Maintaining a decentralized, public ledger with no formal control or governance. There are significant challenges involved.

On the other hand, private distributed ledgers and blockchains can be deployed to solve other sets of problems. As ever, there are tradeoffs and pros and cons to each solution, and you need to consider these individually for each use case.

\section{FUTURE WORK}

Future research can be extended using RFID cards to scan the product information. This project applies to domestic products only. In the future, this can be implemented for product import and export. The traceability for other processed food items can also have the potential for the research. Fresh items like meat, fruits, eggs, seafood, or vegetables are an example of processed food items so research can be implemented on these products also.

\section{REFERENCES}

[1] Attaran M.,12 (4) (2007).,RFID: an enabler of supply chain operations, Suppl. Chain Manag on (Pg 249-257).

[2] Bateman A.H , (6) (2015), Tracking the value of traceability, Suppl. Chain Manag. Rev. 19, (Pg 8-10).

[3] Bello L.L., Mirabella O., Torrisi N., 2005. ETFA 2005. 10th IEEE Conference on, 2, IEEE, 2005., A general approach to model traceability systems in food manufacturing chains, Emerging Technologies and Factory Automation,. (Pg-8)

[4] Berman B., Swani K., (1) (2010), Managing product safety of imported Chinese goods, Bus. Horiz. 53. (Pg 39-48).

[5] Catalini C. (2017). How blockchain technology will impact the digital economy.

[6] Christidis K., Devetsikiotis M., , IEEE Access 4 (2016), Blockchains and smart contracts for the internet of things (Pg 2292-2303).

[7] Deloitte L.L.P., (2016), Blockchain: Enigma, Paradox, Opportunity, The Creative Studio at Deloitte, London J. (Pg. 7969)

[8]. Ellram L.M , (2) (1996), The use of the case study method in logistics research, J. Bus. Logistics 17 (Pg. 93).

[9] Francisco K., Swanson D., (1) (2018). The supply chain has no clothes: technology adoption of blockchain for supply chain transparenc, Logistics 2

[10] Iansiti M., Lakhani K.R., (1) (2017), The truth about blockchain, Harv. Bus. Rev. 95 (Pg 118-127).

[11] Casino Fran, Kanakaris Venetis, Dasaklis Thomas K., Moschuris Socrates, Stachtiaris Spiros, Pagoni Maria \& Rachaniotis Nikolaos P.(2020): Blockchain-based food supply chain traceability: a case study in the dairy sector, International Journal of Production Research, DOI: 10.1080/00207543.2020.1789238.

[12] Shingh Shuvam, Ghimire Sarthak, Kamalvanshi Virendra, Basyal Sudarshan, 2020, Dairy Supply Chain System Based on Blockchain Technology, DOI:10.9734/AJEBA/2020/v14i230189.

[13] ABEYRATNE, S.A. and MONFARED, R.P., 2016. Blockchain ready manufacturing supply chain using distributed ledger. International Journal of Research in Engineering and Technology, 05(09), pp. 1-10.

[14] Wang Yajie, Chen Kai, Hao Miao and Yang Bing, 2020, Food Safety Traceability Method Based on Blockchain Technology, doi:10.1088/1742-6596/1634/1/012025.

[15] Aung M.M., \& Chang Y.S., 2014.Traceability in a food supply chain: Safety and quality presectives. Food Control, pg: 172-184. 